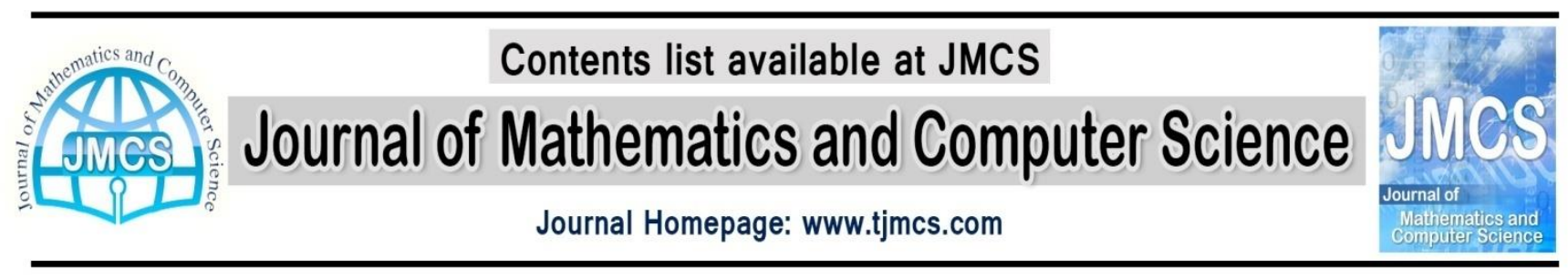

\title{
Numerical Solution of Linear Emden Fowler Boundary Value Problem in Fuzzy Environment
}

\author{
A. F. Jameel ${ }^{1}$, Sarmad A. Altaie ${ }^{2}$ \\ ${ }^{1}$ School of Mathematical Sciences, 11800 USM, University Sains Malaysia, Penang, Malaysia \\ homotopy33@gmail.com \\ ${ }^{2}$ Computer Engineering Department, University of Technology, Baghdad, Iraq \\ altaiesarmad@yahoo.com
}

Article history:

Received January 2015

Accepted April 2015

Available online April 2015

\begin{abstract}
In this paper a numerical method for solving Tow Point Fuzzy Boundary Value Problems '(TPFBVP) involving linear Emden Folwer equation is considered. The finite difference method (FDM) for solving TPFBVP is introduced and the proof of convergence of approximate solutions is brought in detail. Finally a numerical example is solved for illustrating the capability of method.
\end{abstract}

Keywords: Fuzzy numbers, fuzzy differential equations, two point fuzzy boundary value problems, Finite difference method.

\section{Introduction}

Nowadays, fuzzy differential equations (FDEs) [1,2] are a popular topic studied by many researchers since it is utilized widely for the purpose of modeling problems in science and engineering. Most of the practical problems require the solution of a fuzzy differential equation (FDE) which satisfies fuzzy initial or boundary conditions, therefore a fuzzy initial or boundary problem should be solved. However, many fuzzy initial or boundary value problems could not be solved exactly; sometimes it is even impossible to find their analytical solutions. Thus, considering their approximate solutions is becoming more important. The two-point boundary value problem (TPBVP) occurs in a wide variety of problems in engineering [3, 4] and science, including the modeling of chemical reactions [5, 6], heat transfer [7,8], and diffusion $[9,10]$, and the solution of optimal control problems [11,12]. Fuzzy two point boundary value problems (FTPBVP) appears when the modeling of these problems cannot be sure is perfect and its nature is under uncertainty. Fuzzy ordinary differential equations are suitable mathematical models to model dynamical systems in which there exist uncertainties or vagueness. These models are used in various applications including population models [13], quantum optics gravity [14], and medicine [15, 16]. Fuzzy two point boundary value problems have been solved using FDM in [17] for linear problems with fuzzy boundary conditions, also a initial value method in [18] are applied to get the numerical solution for linear TPFBVP .The Finite Difference Method (FDM) is one of the simplest and of the oldest methods to linear TPFBVP 
solve differential equations. It consists in approximating the differential operator by replacing the derivatives in the equation using differential quotients. The domains is partitioned in space and in time and approximations of the solution are computed at the space or time .To the best of our knowledge, this is the first attempt for solving linear fuzzy Emden Folwer. The structure of this paper is as follows. In section 2, some basic definitions and notations are given which will be used in other sections. In section 3 , the defuzzification of TPFBVP is given in details. In section 4, structure of FDM is formulated for solving TPFBVP. In section 5, the definitions and thermos of consistency, stability and convergence related with FDM in section 4 are presented in details. In section 6, we introduced linear Emden Folwer in fuzzy environment with the proof the uniqueness of the difference approximation. In section 7 a numerical example is solved for illustrating of the method and finally, in section 8 , we give the conclusion of this study.

\section{Preliminaries}

The definitions reviewed in this section are required in our work.

Definition 1 [19, 20]: Let $\tilde{E}$ be the set of all upper semi-continuous normal convexfuzzy numbers with $r$ level bounded intervals such that:

$[\mu]_{r}=\{t \in \mathbb{R}: \mu \geq r\}$.

An arbitrary fuzzy number is represented by an ordered pair of functions

$[\tilde{\mu}(t)]_{r}=[\underline{\mu}(t), \bar{\mu}(t)]_{r}$ for all $r \in[0,1]$ which satisfies:

1. $\mu(t)$ is normal, i.e $\exists t_{0} \in \mathbb{R}$ with $\mu\left(t_{0}\right)=1$.

2. $\mu(t)$ is convex fuzzy set, i.e. $\mu(\lambda t+(1-\lambda) s) \geq \min \{\mu(t), \mu(s)\}, \forall t, s \in \mathbb{R}, \lambda \in[0,1]$.

3. $[\tilde{\mu}(t)]_{r} \in \tilde{E}, \mu$ is upper semi continuous on $\mathbb{R}$.

4. $\{t \in \mathbb{R}: \mu(t)>0\}$ is compact.

5. $\mu(t)$ is a bounded left continuous non-decreasing function over $[0,1]$.

6. $\overline{\bar{\mu}}(t)$ is a bounded left continuous non-increasing function over[0,1].

7. $\mu(t) \leq \bar{\mu}(t)$, for all $r \in[0,1]$.

The $r$-level sets of a fuzzy number are much more effective as representation forms of fuzzy set than the above. Fuzzy sets can be defined by the families of their $r$-level sets based on the resolution identity theorem [21].

Definition 2.1 [22]: A mapping $\tilde{f}: T \rightarrow \widetilde{E}$ (or $\widetilde{P}(E)$ ) for some interval $T \subseteq \widetilde{E}$ iscalledafuzzy process or fuzzy function with crisp variable, and we denote $r$-level set by:

$$
[\tilde{f}(t)]_{r}=[\underline{f}(t ; r), \bar{f}(t ; r)], t \in T, r \in[0,1]
$$

where $\tilde{E}$ be the set of all upper semi-continuous normal convexfuzzy numbers.

Definition 2.2 [23, 24]: Each function $f: X \rightarrow Y$ induces another function $\tilde{f}: F(X) \rightarrow F(Y)$ defined for each fuzzy interval $U$ in $X$ by:

$$
\tilde{f}(U)(y)= \begin{cases}\operatorname{Sup}_{x \in f^{-1}(y)} U(x), & \text { if } y \in \operatorname{range}(f) \\ 0 & \text {, if } y \notin \operatorname{range}(f)\end{cases}
$$

This is called the Zadeh extension principle. 
Definition 2.3 [25]: A fuzzy matrix of order $m \times s$ is defined $[\tilde{A}]=\left[\tilde{a}_{i j}, \mu_{\tilde{a}_{i j}}\right]$ as, where $\mu_{\tilde{a}_{i j}}$ is the membership function of the element $\tilde{a}_{i j} \operatorname{in}[\tilde{A}], \forall \tilde{a}_{i j} \in \tilde{E}$, for $i=1,2, \ldots m, j=1,2, \ldots . . s$.Thus for all $r \in[0,1]$

$[\tilde{A}]_{r}=[\underline{A}, \bar{A}]_{r}$, and $\left[\tilde{a}_{i j}\right]_{r}=\left[\underline{a}_{i j}, \bar{a}_{i j}\right]_{r}$.

\section{Defuzzification of FTPBVP}

Consider the following second order FTPBVP

$$
\left\{\begin{array}{c}
\tilde{y}^{\prime \prime}(x)=\tilde{f}\left(x, \tilde{y}(x), \tilde{y}^{\prime}(x)\right), x \in\left[x_{0}, x_{n}\right] \\
\tilde{y}\left(x_{0}\right)=\tilde{\alpha}, \tilde{y}\left(x_{n}\right)=\tilde{\beta}
\end{array}\right.
$$

According to Section 2, It's not difficult for defuzzification of Eq. (1), where $\tilde{y}(x)$ is a fuzzy function of the crisp variable $x$ and $\tilde{f}$ is the fuzzy function of the crisp variable $x$ and the fuzzy variable $\tilde{y}$. Here $\tilde{y}^{\prime \prime}(x)$ is the second order fuzzy derivative [26] of $\tilde{y}(x), \tilde{y}^{\prime}(x)$, with $\tilde{\alpha}, \tilde{\beta}$ are the fuzzy numbers that refer to the fuzzy boundary conditions $\tilde{y}\left(x_{0}\right), \tilde{y}\left(x_{n}\right)$ of Eq.(1). We denote the fuzzy function $\tilde{y}(x)$ by $[\tilde{y}]_{r}=$ $[\underline{y}, \bar{y}]_{r}$, for $x \in\left[x_{0}, x_{n}\right]$ and $r \in[0,1]$ it means that the $r$-level set of $\tilde{y}(x)$ can be defined as:

$$
\begin{aligned}
& \left\{\begin{array}{l}
{[\tilde{y}(x)]_{r}=[\underline{y}(x ; r), \bar{y}(x ; r)]} \\
{\left[\tilde{y}^{\prime}(x)\right]_{r}=\left[\underline{y}^{\prime}(x ; r), \bar{y}^{\prime}(x ; r)\right]} \\
{\left[\tilde{y}^{\prime \prime}(x)\right]_{r}=\left[\underline{y}^{\prime \prime}(x ; r), \bar{y}^{\prime \prime}(x ; r)\right]}
\end{array}\right. \\
& \left\{\begin{array}{l}
{\left[\tilde{y}\left(x_{0}\right)\right]_{r}=\left[\underline{y}\left(x_{0} ; r\right), \bar{y}\left(x_{0} ; r\right)\right]=[\underline{\alpha}, \bar{\alpha}]_{r}} \\
{\left[\tilde{y}\left(x_{n}\right)\right]_{r}=\left[\underline{y}\left(x_{n} ; r\right), \bar{y}\left(x_{n} ; r\right)\right]=[\underline{\beta}, \bar{\beta}]_{r}}
\end{array}\right.
\end{aligned}
$$

Now if we let $\mathcal{Y}(x)=y(x), y^{\prime}(x)$, and for defuzzification we have

$$
\tilde{y}(x ; r)=[\underline{y}(x ; r), \bar{y}(x ; r)]
$$

where

$$
\begin{aligned}
& \underline{y}(x ; r)=\left[\underline{y}(x ; r), \underline{y}^{\prime}(x ; r), \underline{y}^{\prime \prime}(x ; r)\right] \\
& \bar{y}(x ; r)=\left[\bar{y}(x ; r), \bar{y}^{\prime}(x ; r), \bar{y}^{\prime \prime}(x ; r)\right]
\end{aligned}
$$

Also we can write

$$
[\tilde{f}(x, \tilde{\mathcal{Y}})]_{r}=[\underline{f}(x, \tilde{\mathcal{Y}} ; r), \bar{f}(x, \tilde{\mathcal{Y}} ; r)]
$$

and by using the extension fuzzy principle we have

$$
\left\{\begin{array}{l}
\underline{f}(x, \tilde{\mathcal{y}} ; r)=\mathcal{F}[x, \underline{y}, \overline{\mathcal{y}}]_{r}, \\
\bar{f}(x, \tilde{\mathcal{y}} ; r)=\mathcal{G}[x, \underline{\mathcal{y}}, \overline{\mathcal{y}}]_{r}
\end{array}\right.
$$


Since $\tilde{y}^{\prime \prime}(x)=f(x, y(x))$, we can define the following membership function

$$
\left\{\begin{array}{l}
\underline{f}(x, \tilde{\mathcal{Y}}(x ; r) ; r)=\min \{\tilde{f}(x, \tilde{\mu}(r)) \mid \tilde{\mu}(r) \in \tilde{\mathcal{Y}}(t ; r)\} \\
\bar{f}(x, \tilde{\mathcal{Y}}(x ; r) ; r)=\max \{\tilde{f}(x, \tilde{\mu}(r)) \mid \tilde{\mu}(r) \in \tilde{\mathcal{Y}}(t ; r)\}
\end{array}\right.
$$

where

$$
\left\{\begin{array}{l}
\underline{f}(x, \tilde{\mathcal{Y}}(x ; r) ; r)=\mathcal{F}(x, \underline{y}(x ; r), \overline{\mathcal{Y}}(x ; r)) \\
\bar{f}(x, \tilde{\mathcal{Y}}(x ; r) ; r)=\mathcal{G}(x, \underline{\mathcal{Y}}(x ; r), \overline{\mathcal{Y}}(x ; r))
\end{array}\right.
$$

After this defuzzification can rewrite Eq. (1) for the lower and the upper bound of respectively for Eq. (1) and for all $r \in[0,1]$, we have

$$
\begin{gathered}
\left\{\begin{array}{c}
\underline{y}^{\prime \prime}(x ; r)=\mathcal{F}(x, \underline{\mathcal{Y}}(x ; r), \overline{\mathcal{Y}}(x ; r)), x \in\left[x_{0}, x_{n}\right] \\
\underline{y}\left(x_{0} ; r\right)=[\underline{\alpha}]_{r}, \underline{y}\left(x_{n} ; r\right)=[\underline{\beta}]_{r}
\end{array}\right. \\
\left\{\begin{array}{c}
\bar{y}^{\prime \prime}(x ; r)=\mathcal{G}(x, \underline{\mathcal{Y}}(x ; r), \overline{\mathcal{Y}}(x ; r)), x \in\left[x_{0}, x_{n}\right] \\
\bar{y}\left(x_{0} ; r\right)=[\bar{\alpha}]_{r}, \bar{y}\left(x_{n} ; r\right)=[\bar{\beta}]_{r}
\end{array}\right.
\end{gathered}
$$

More details about the existence and uniqueness are in [17].

\section{Finite Difference Scheme for Second Order Linear FTPBVP}

It consists in approximating the differential operator by replacing the derivatives in the equation using differential quotients. The domain is partitioned in space and in time and approximations of the solution are computed at the space or time points. The error between the numerical solution and the exact solution is determined by the error that is committed by going from a differential operator to a difference operator. This error is called the called the truncation error [9]. The term truncation error reflects the fact that a finite part of a Taylor series is used in the approximation. We formulate this scheme in order to solve second order TPBVP in fuzzy environment. We consider fuzzy boundary value Eq. (1) can be written as:

$$
\left\{\begin{array}{c}
\tilde{y}^{\prime \prime}(x)=p(x) \tilde{y}^{\prime}(x)+q(x) \tilde{y}(x)+\widetilde{w}(x), x \in\left[x_{0}, x_{n}\right] \\
y\left(x_{0}\right)=\tilde{\alpha}, y\left(x_{n}\right)=\tilde{\beta}
\end{array}\right.
$$

Where $p(x), q(x)$ and $w(x)$ are real continuous functions on $J=\left[x_{0}, x_{n}\right]$ with $\tilde{\alpha}, \tilde{\beta} \in \tilde{E}$, then according to Section 2 and for all $r \in[0,1]$, we have the same defuzzification of Eq. (1) of $\tilde{y}(x), \widetilde{w}(x), \tilde{y}^{\prime}(x), \tilde{y}^{\prime \prime}(x), \tilde{y}\left(x_{0}\right), \tilde{y}\left(x_{n}\right)$.

\subsection{Approximation of the First Order Derivative}

In this section we derive a finite difference formula for the fuzzy derivative $\left[\tilde{y}^{\prime}(x)\right]_{r}$ with its accuracy analysis. Suppose the function $[\tilde{y}(x)]_{r} \in C^{2}(\tilde{E})$ continuous for all $x \in J$. Using Taylor series for any $h>0$ we have the forward difference formula: 


$$
\tilde{y}(x+h ; r)=\tilde{y}(x ; r)+h \tilde{y}^{\prime}(x ; r)+\frac{h^{2}}{2} \tilde{y}^{\prime \prime}(x ; r)+\frac{h^{3}}{6} \tilde{y}^{\prime \prime \prime}(x ; r)+\frac{h^{4}}{24} \tilde{y}^{(4)}\left(\zeta^{+} ; r\right)
$$

where $\zeta^{+} \in[x+h, x]$, and backward difference formula:

$$
\tilde{y}(x-h ; r)=\tilde{y}(x ; r)-h \tilde{y}^{\prime}(x ; r)+\frac{h^{2}}{2} \tilde{y}^{\prime \prime}(x ; r)-\frac{h^{3}}{6} \tilde{y}^{\prime \prime \prime}(x ; r)+\frac{h^{4}}{24} \tilde{y}^{(4)}\left(\zeta^{-} ; r\right)
$$

where $\zeta^{-} \in[x-h, x]$.By subtracting (3.4) from (3.5) we have fuzzy central difference formula :

$$
\tilde{y}^{\prime}(x ; r)=\frac{\tilde{y}(x+h ; r)-\tilde{y}(x-h ; r)}{2 h}+O\left(h^{2}\right)
$$

where $O\left(h^{2}\right)=-\frac{h^{3}}{3} \tilde{y}^{\prime \prime \prime}(\zeta ; r)$, and $\zeta \in[x-h, x+h]$. Hence, for every $h \in\left[0, h_{0}\right]$, we have the following bound on the approximation truncation error:

where

$$
\left|\frac{\tilde{y}(x+h ; r)-\tilde{y}(x-h ; r)}{2 h}\right| \leq \tilde{C} h^{2}
$$

$$
\begin{aligned}
& \underline{C}=\sup _{\zeta \in[x-h, x+h]}\left\{\min \left(\frac{h^{3}}{3} \underline{y}^{\prime \prime \prime}(\zeta ; \mu), \frac{h^{3}}{3} \bar{y}^{\prime \prime \prime}(\zeta ; \mu) \mid \mu \in[\underline{y}(\zeta), \bar{y}(\zeta)]_{r}\right)\right\} \\
& \bar{C}=\sup _{\zeta \in[x-h, x+h]}\left\{\max \left(\frac{h^{3}}{3} \underline{y}^{\prime \prime \prime}(\zeta ; \mu), \frac{h^{3}}{3} \bar{y}^{\prime \prime \prime}(\zeta ; \mu) \mid \mu \in[\underline{y}(\zeta), \bar{y}(\zeta)]_{r}\right)\right\}
\end{aligned}
$$

Here $\mu$ represent the membership function of the FTPBVP (10) for all $r \in[0,1]$ and $h$ is the step size given by $h=x_{n}-x_{0} / N$, where $N$ is the number of iterative.

\subsection{Approximation of the Second Order Derivative}

In this section we derive a finite difference formula for $\tilde{y}^{\prime \prime}(x ; r)$ with its accuracy analysis. Suppose the function $[\tilde{y}(x)]_{r} \in C^{4}(\tilde{E})$ continuous for all $\mathrm{x} \in J$. Using the Taylor series for any $h>0$ and by adding formula (3.4) from (3.5) we have central fuzzy difference formula:

$$
\tilde{y}^{\prime \prime}(x ; r)=\frac{\tilde{y}(x+h ; r)-2 \tilde{y}(x ; r)+\tilde{y}(x-h ; r)}{h^{2}}+O\left(h^{2}\right)
$$

where $O\left(h^{2}\right)=-\frac{h^{4}}{12} \tilde{y}^{(4)}(\zeta ; r), \zeta \in[x-h, x+h]$. Hence, for every $h \in\left[0, h_{0}\right]$, we have the following bound on the approximation truncation error:

$$
\left|\frac{\tilde{y}(x+h ; r)-2 \tilde{y}(x ; r)+\tilde{y}(x-h ; r)}{h^{2}}\right| \leq \tilde{C} h^{2}
$$

where

$$
\begin{aligned}
& \underline{C}=\sup _{\zeta \in[x-h, x+h]}\left\{\min \left(\frac{h^{4}}{12} \underline{y}^{(4)}(\zeta ; \mu), \frac{h^{4}}{12} \bar{y}^{(4)}(\zeta ; \mu) \mid \mu \in[\underline{y}(\zeta), \bar{y}(\zeta)]_{r}\right)\right\} \\
& \bar{C}=\sup _{\zeta \in[x-h, x+h]}\left\{\max \left(\frac{h^{4}}{12} \underline{y}^{(4)}(\zeta ; \mu), \frac{h^{4}}{12} \bar{y}^{(4)}(\zeta ; \mu) \mid \mu \in[\underline{y}(\zeta), \bar{y}(\zeta)]_{r}\right)\right\}
\end{aligned}
$$

Here $\mu$ represent the membership function of the FTPBVP (10) for all $r \in[0,1]$ and $h$ is the step size given by $h=x_{n}-x_{0} / N$, where $N$ is the number of iterations. In our work we set 
$\tilde{y}(x+h ; r)=\left[\tilde{y}_{i+1}\right]_{r}, \tilde{y}(x-h ; r)=\left[\tilde{y}_{i-1}\right]_{r}$, and $\tilde{y}(x ; r)=\left[\tilde{y}_{i}\right]_{r}$ for $i=1,2 \ldots n$ for arbitrary positive integer $n$ we subdivide the interval $J=\left[x_{0}, x_{n}\right]$ as $x_{0}<x_{1}<\cdots<x_{n-1}<x_{n}$. Let $\pi=\left\{x_{i}\right\}_{i=0}^{n+1}$ denote a uniform partition of the interval $J$ such that $x_{i}=x_{0}+i h, i=0,1, \ldots n+1$, with $h=x_{n}-x_{0} / n+1$, and for all $r \in[0,1]$ we can rewrite the fuzzy central difference formula as:

$$
\left\{\begin{array}{l}
{\left[\tilde{y}_{i}{ }^{\prime}\right]_{r}=\frac{\left[\tilde{y}_{i+1}\right]_{r}-\left[\tilde{y}_{i-1}\right]_{r}}{2 h}} \\
{\left[\tilde{y}_{i}{ }^{\prime \prime}\right]_{r}=\frac{\left[\tilde{y}_{i+1}\right]_{r}-2\left[\tilde{y}_{i}\right]_{r}+\left[\tilde{y}_{i-1}\right]_{r}}{h^{2}}}
\end{array}\right.
$$

The Uniqueness of the Difference Approximation of Eq. (10) is given in [15]

\section{Consistency, Stability and Convergence}

To study the accuracy and the computability of the difference approximation in fuzzy environment, such that $\left\{\tilde{y}_{i+1}\right\}_{i=0}^{n}$, we introduce the concepts of consistency, stability and convergence of finite difference methods. The basic result proved in this section is that, for a consistent method, stability implies convergence.

Definition 5.1 (Consistency): Let

$$
\left[\tilde{\mathcal{T}}_{i, \pi}\right]_{r}=\tilde{L}_{h} \tilde{v}\left(x_{i} ; r\right)-\tilde{L} \tilde{v}\left(x_{i} ; r\right), i=1,2, \ldots, n
$$

where $\tilde{v}$ is smooth and continuous fuzzy function on $\tilde{E}, \forall x \in J, \tilde{L} h$ is the difference operator and $\tilde{L}$ is the linear operator of Eq. (10).Then the difference problem (15) is consistent with the differential problem (10) if:

$$
\left[\tilde{\mathcal{T}}_{i, \pi}(\tilde{v})\right]_{r} \rightarrow 0 \text { as } h \rightarrow 0
$$

Where $\left[\tilde{\mathcal{T}}_{i, \pi}(\tilde{v})\right]_{r}$ refer to local truncation (or local discretization) errors.

Definition 5.2: The difference problem (15) is locally $\mathrm{p}^{\text {th }}$ order accurate if sufficiently smooth data, there exists a positive constant $[\tilde{C}]_{r}=[\underline{C}, \bar{C}]_{r}$, independent of $h$, such that:

$$
\begin{aligned}
& \sup _{1 \leq i \leq n}\left\{\min \left|\left[\underline{\mathcal{T}}_{i, \pi}(\underline{v})\right]_{r},\left[\overline{\mathcal{T}}_{i, \pi}(\bar{v})\right]_{r}\right|\right\} \leq[\underline{C}]_{r} h^{p} \\
& \sup _{1 \leq i \leq n}\left\{\max \left|\left[\underline{\mathcal{T}}_{i, \pi}(\underline{v})\right]_{r},\left[\overline{\mathcal{T}}_{i, \pi}(\bar{v})\right]_{r}\right|\right\} \leq[\bar{C}]_{r} h^{p}
\end{aligned}
$$

The following lemma demonstrates that the difference problem (15) is consistent with (10) and is locally second order accurate.

Lemma 5.1: If $\tilde{v} \in C^{4}(J)$ then

$$
\begin{gathered}
{\left[\tilde{\mathcal{T}}_{i, \pi}(\tilde{v})\right]_{r}=} \\
\quad \frac{-h^{2}}{12}\left(\tilde{v}^{(4)}\left(\tau_{i} ; r\right)-2 p\left(x_{i}\right) \tilde{v}^{\prime \prime \prime}\left(\theta_{i} ; r\right)\right) \\
\forall r \in[0,1], i=1,2, \ldots, n
\end{gathered}
$$

$\tau_{i}, \theta_{i}$ lie in $\left(x_{i-1}, x_{i+1}\right)$ 


\section{Proof:}

According to definition (5.2) one can write

$$
\left[\widetilde{\mathcal{T}}_{i, \pi}(\widetilde{v})\right]_{r}=\left[\frac{\widetilde{v}\left(x_{i+1} ; r\right)-2 \widetilde{v}\left(x_{i} ; r\right)+\widetilde{v}\left(x_{i-1} ; r\right)}{h^{2}}-\widetilde{v}^{\prime \prime}\left(x_{i} ; r\right)\right]+p\left(x_{i}\right)\left[\frac{\widetilde{v}\left(x_{i+1} ; r\right)-\widetilde{v}\left(x_{i-1} ; r\right)}{2 h}-\tilde{v}^{\prime}\left(x_{i} ; r\right)\right]
$$

It is easy to show using Taylor's the theorem that:

$$
\frac{\widetilde{v}\left(x_{i+1} ; r\right)-\widetilde{v}\left(x_{i-1} ; r\right)}{2 h}-\tilde{v}^{\prime}\left(x_{i} ; r\right)=\frac{h^{2}}{3} \tilde{v}^{\prime \prime \prime}\left(\theta_{i} ; r\right)
$$

$\theta_{i} \in\left(x_{i-1}, x_{i+1}\right)$

$$
\frac{\widetilde{v}\left(x_{i+1} ; r\right)-2 \widetilde{v}\left(x_{i} ; r\right)+\widetilde{v}\left(x_{i-1} ; r\right)}{h^{2}}-\widetilde{v}^{\prime \prime}\left(x_{i} ; r\right)=\frac{h^{2}}{12} \widetilde{v}^{(4)}\left(\tau_{i} ; r\right)
$$

$\tau_{i} \in\left(x_{i-1}, x_{i+1}\right)$

The desired result now follows on substituting (17) and (18) in (16).

Definition 5.3 (Stability): The linear difference operator $\tilde{L}_{h}$ is stable if, for sufficiently small $h$, there exists a constant $K$, independent of $h$, such that

$$
\begin{aligned}
& \left.\left|\left[\underline{v}_{i}\right]_{r}\right| \leq K\left\{\sup \left\{\min \left(\left(\left(\mid \underline{v}_{0}\right]_{r}|,|\left[\underline{v}_{n+1}\right]_{r} \mid\right),\left|\left[\bar{v}_{0}\right]_{r}\right|,\left|\left[\bar{v}_{n+1}\right]_{r}\right|\right)\right)\right\}\right\}+\sup _{1 \leq j \leq n}\left\{\min \left(\left[\underline{L}_{h} \underline{v}_{j}\right]_{r},\left[\bar{L}_{h} \bar{v}_{j}\right]_{r}\right)\right\} \\
& \left|\left[\bar{v}_{i}\right]_{r}\right| \leq K\left\{\sup \left\{\max \left(\left(\left(\left|\left[\underline{v}_{0}\right]_{r}\right|,\left|\left[\underline{v}_{n+1}\right]_{r}\right|\right),\left|\left[\bar{v}_{0}\right]_{r}\right|,\left|\left[\bar{v}_{n+1}\right]_{r}\right|\right)\right)\right\}\right\}+\sup _{1 \leq j \leq n}\left\{\max \left(\left[\underline{L}_{h} \underline{v}_{j}\right]_{r},\left[\bar{L}_{h} \bar{v}_{j}\right]_{r}\right)\right\} \\
& \forall r \in[0,1], i=0,1, \ldots, n+1 .
\end{aligned}
$$

We now prove that, for $h$ sufficiently small, the difference operator $\tilde{L}_{h}$ of (16) is stable.

Theorem 5.1: If the functions $p$ and q satisfy ( ), then the difference operator $\tilde{L}_{h}$ of Eq. (4.1) is stable for, $h<\min \left(2 / p^{*}, p_{*} / q^{*}\right)$ with $K=\left\{1,1 / q_{*}\right\}$

Proof: If

$$
\begin{aligned}
& \left|\left[\underline{v}_{i}\right]_{r}\right| \leq \sup _{1 \leq j \leq n}\left\{\min \left(\left|\left[\underline{v}_{j}\right]_{r}\right|,\left|\left[\bar{v}_{j}\right]_{r}\right|\right)\right\} \\
& \left|\left[\bar{v}_{i}\right]_{r}\right| \leq \sup _{1 \leq j \leq n}\left\{\min \left(\left|\left[\underline{v}_{j}\right]_{r}\right|,\left|\left[\bar{v}_{j}\right]_{r}\right|\right)\right\}
\end{aligned}
$$

$\forall r \in[0,1], i=0,1, \ldots, n+1$.

then from (4.1) we obtain

$$
d_{i}\left[\underline{v}_{i}\right]_{r} \leq\left(\left|e_{i}\right|+\left|c_{i}\right|\right)\left|\left[\underline{v}_{j}\right]_{r}\right|+h^{2} \sup _{1 \leq j \leq n}\left\{\min \left(\left|\left[\underline{L}_{h} \underline{v}_{j}\right]_{r}\right|,\left|\left[\bar{L}_{h} \bar{v}_{j}\right]_{r}\right|\right)\right\}
$$




$$
d_{i}\left[\bar{v}_{i}\right]_{r} \leq\left(\left|e_{i}\right|+\left|c_{i}\right|\right)\left|\left[\bar{v}_{j}\right]_{r}\right|+h^{2} \sup _{1 \leq j \leq n}\left\{\max \left(\left|\left[\underline{L}_{h} \underline{v}_{j}\right]_{r}\right|,\left|\left[\bar{L}_{h} \bar{v}_{j}\right]_{r}\right|\right)\right\}
$$

then

$$
d_{i}\left[\tilde{v}_{i}\right]_{r}=-e_{i}\left[\tilde{v}_{i+1}\right]_{r}-c_{i}\left[\tilde{v}_{i-1}\right]_{r}+h^{2}\left[\tilde{L}_{h} \tilde{v}_{i+1}\right]_{r}
$$

Thus

if $h<\min \left(2 / p^{*}, p_{*} / q^{*}\right)$, then

$$
d_{i}=\left|c_{i}\right|+\left|e_{i}\right|+h^{2} q_{i}
$$

and it follows that

$$
\begin{aligned}
& h^{2} q_{i}\left|\left[\underline{v}_{i}\right]_{r}\right| \leq h^{2} \sup _{1 \leq j \leq n}\left\{\min \left(\left|\left[\underline{L}_{h} \underline{v}_{j}\right]_{r}\right|,\left|\left[\bar{L}_{h} \bar{v}_{j}\right]_{r}\right|\right)\right\} \\
& h^{2} q_{i}\left|\left[\bar{v}_{i}\right]_{r}\right| \leq h^{2} \sup _{1 \leq j \leq n}\left\{\max \left(\left|\left[\underline{L}_{h} \underline{v}_{j}\right]_{r}\right|,\left|\left[\bar{L}_{h} \bar{v}_{j}\right]_{r}\right|\right)\right\}
\end{aligned}
$$

or

$$
\begin{aligned}
& \left|\left[\underline{v}_{i}\right]_{r}\right| \leq \frac{1}{q^{*}} \sup _{1 \leq j \leq n}\left\{\min \left(\left|\left[\underline{L}_{h} \underline{v}_{j}\right]_{r}\right|,\left|\left[\bar{L}_{h} \bar{v}_{j}\right]_{r}\right|\right)\right\} \\
& \left|\left[\bar{v}_{i}\right]_{r}\right| \leq \frac{1}{q^{*}} \sup _{1 \leq j \leq n}\left\{\max \left(\left|\left[\underline{L}_{h} \underline{v}_{j}\right]_{r}\right|,\left|\left[\bar{L}_{h} \bar{v}_{j}\right]_{r}\right|\right)\right\}
\end{aligned}
$$

Thus if $\max x_{0} \leq i \leq x_{n+1}\left[\tilde{v}_{i+1}\right]_{r}$ occurs for $1 \leq j \leq n$, then

$$
\begin{aligned}
& \sup _{0 \leq i \leq n+1}\left\{\min \left(\left|\left[\underline{v}_{i}\right]_{r}\right|,\left|\left[\bar{v}_{i}\right]_{r}\right|\right)\right\} \leq \frac{1}{q^{*}} \sup _{1 \leq j \leq n}\left\{\min \left(\left|\left[\underline{L}_{h} \underline{v}_{j}\right]_{r}\right|,\left|\left[\bar{L}_{h} h \bar{v}_{j}\right]_{r}\right|\right)\right\} \\
& \sup _{1 \leq i \leq n+1}\left\{\max \left(\left|\left[\underline{v}_{i}\right]_{r}\right|,\left|\left[\bar{v}_{i}\right]_{r}\right|\right)\right\} \leq \frac{1}{q^{*}} \sup _{1 \leq j \leq n}\left\{\max \left(\left|\left[\underline{L}_{h} \underline{v}_{j}\right]_{r}\right|,\left|\left[\bar{L}_{h} \bar{v}_{j}\right]_{r}\right|\right)\right\}
\end{aligned}
$$

and clearly

$$
\begin{aligned}
& \sup _{0 \leq i \leq n+1}\left\{\min \left(\left|\left[\underline{v}_{i}\right]_{r}\right|,\left|\left[\bar{v}_{i}\right]_{r}\right|\right)\right\} \leq \\
& K\left\{\sup \left\{\min \left(\left(\left|\left[\underline{v}_{0}\right]_{r}\right|,\left|\left[\underline{v}_{n+1}\right]_{r}\right|\right),\left(\left|\left[\bar{v}_{0}\right]_{r}\right|,\left|\left[\bar{v}_{n+1}\right]_{r}\right|\right)\right)\right\}\right\}+\sup _{1 \leq j \leq n}\left\{\min \left(\left|\left[\underline{L}_{h} \underline{v}_{j}\right]_{r}\right|,\left|\left[\bar{L}_{h} \bar{v}_{j}\right]_{r}\right|\right)\right\} \\
& \sup _{1 \leq i \leq n+1}\left\{\max \left(\left|\left[\underline{v}_{i}\right]_{r}\right|,\left|\left[\bar{v}_{i}\right]_{r}\right|\right)\right\} \leq K\left\{\sup \left\{\max \left(\left(\left|\left[\underline{v}_{0}\right]_{r}\right|,\left|\left[\underline{v}_{n+1}\right]_{r}\right|\right),\left(\left|\left[\bar{v}_{0}\right]_{r}\right|,\left|\left[\bar{v}_{n+1}\right]_{r}\right|\right)\right)\right\}\right\}+ \\
& \sup _{1 \leq j \leq n}\left\{\max \left(\left|\left[\underline{L}_{h} \underline{v}_{j}\right]_{r}\right|,\left|\left[\bar{L}_{h} \bar{v}_{j}\right]_{r}\right|\right)\right\}
\end{aligned}
$$

Thus if $\max x_{0} \leq i \leq x_{n+1}\left|\left[\tilde{v}_{i}\right]_{r}\right|=\sup \left\{\left|\left[\tilde{v}_{0}\right]_{r}\right|,\left|\left[\tilde{v}_{n+1}\right]_{r}\right|\right\}$ such that

$$
\left|\left[\underline{v}_{i}\right]_{r}\right|=\sup \left\{\min \left(\left(\left|\left[\underline{v}_{0}\right]_{r}\right|,\left|\left[\underline{v}_{n+1}\right]_{r}\right|\right),\left(\left|\left[\bar{v}_{0}\right]_{r}\right|,\left|\left[\bar{v}_{n+1}\right]_{r}\right|\right)\right)\right\}
$$




$$
\left|\left[\bar{v}_{i}\right]_{r}\right|=\sup \left\{\max \left(\left(\left|\left[\underline{v}_{0}\right]_{r}\right|,\left|\left[\underline{v}_{n+1}\right]_{r}\right|\right),\left(\left|\left[\bar{v}_{0}\right]_{r}\right|,\left|\left[\bar{v}_{n+1}\right]_{r}\right|\right)\right)\right\}
$$

then the above equation follows immediately. An immediate consequence of stability is the uniqueness (and hence existence since the problem is linear) of the difference approximation $\left\{\left[\tilde{y}_{i}\right]_{r}\right\}_{i=0}^{n}$, for, if they were two solutions, their difference $\left\{\left[\tilde{v}_{i}\right]_{r}\right\}_{i=0}^{n}$, say, would satisfy:

$$
\begin{gathered}
\left|\left[\tilde{L}_{h} \tilde{v}_{j}\right]_{r}\right|=0, j=1,2, \ldots, n \\
{\left[\tilde{v}_{0}\right]_{r}=\left[\tilde{v}_{n+1}\right]_{r}=0}
\end{gathered}
$$

Stability then implies that $\left[\tilde{v}_{i}\right]_{r}=0$ for $i=0,1,2, \ldots, n+1$.

Definition 5.4(Convergence): Let $\tilde{Y}(x ; r)$ be the exact solution of the boundary value Eq. (10), and $\left\{\left[\tilde{y}_{i}\right]_{r}\right\}_{i=0}^{n}$ be the difference approximation defined by Eq. (15). The difference approximation converges to $\tilde{Y}(x ; r)$ if

$$
\max _{1 \leq j \leq n}\left|\tilde{Y}\left(x_{j} ; r\right)-\tilde{y}\left(x_{j} ; r\right)\right| \rightarrow 0
$$

or

$$
\begin{aligned}
& {[\underline{\mathbb{E}}]_{r}=\sup _{1 \leq j \leq n}\left\{\min \left(\left|\underline{Y}\left(x_{j} ; r\right)-\underline{y}\left(x_{j} ; r\right)\right|,\left|\bar{Y}\left(x_{j} ; r\right)-\underline{y}\left(x_{j} ; r\right)\right|\right)\right\} \rightarrow 0} \\
& {[\overline{\mathbb{E}}]_{r}=\sup _{1 \leq j \leq n}\left\{\max \left(\left|\underline{Y}\left(x_{j} ; r\right)-\underline{y}\left(x_{j} ; r\right)\right|,\left|\bar{Y}\left(x_{j} ; r\right)-\underline{y}\left(x_{j} ; r\right)\right|\right)\right\} \rightarrow 0}
\end{aligned}
$$

$\forall r \in[0,1]$,

as $h \rightarrow 0$. The difference $\tilde{Y}\left(x_{j} ; r\right)-\tilde{y}\left(x_{j} ; r\right)$ is the global truncation (or discretization) error $[\widetilde{\mathbb{E}}]_{r}=$ $[\underline{\mathbb{E}}, \mathbb{E}]_{r}$ at the point $x_{j}, j=1,2, \ldots, n$

Definition 5.9: The difference approximation $\left\{\left[\tilde{y}_{i}\right]_{r}\right\}_{i=0}^{n}$ is a $p^{\text {th }}$ approximation to the solution $\tilde{Y}(x ; r)$ if for $h$ sufficiently small; there exists a constant $[\tilde{\mathcal{C}}]_{r}$ independent of $h$ such that

$$
\max _{1 \leq j \leq n}\left|\tilde{Y}\left(x_{j} ; r\right)-\tilde{y}\left(x_{j} ; r\right)\right| \leq[\tilde{\mathcal{C}}]_{r} h^{p}
$$

The basic result connecting consistency, stability and convergence is given in the following theorem.

Theorem 5.2: Suppose $\tilde{Y}(x ; r) \in C^{4}(J)$, and $h<\frac{2}{p^{*}}$, Then the difference solution $\left\{\left[\tilde{y}_{i}\right]_{r}\right\}_{i=0}^{n}$ of (2.1) is convergent to the $\tilde{Y}(x ; r)$ solution of Eq. (15) Moreover,

where

$$
\max _{1 \leq j \leq n}\left|\tilde{Y}\left(x_{j} ; r\right)-\tilde{y}\left(x_{j} ; r\right)\right| \leq[\tilde{\mathcal{C}}]_{r} h^{2}
$$

$$
\begin{aligned}
& \underline{\mathcal{C}}=\sup _{\zeta \in[x-h, x+h]}\left\{\min \left(\frac{h^{4}}{12} \underline{y}^{(4)}(\zeta ; \mu), \frac{h^{4}}{12} \bar{y}^{(4)}(\zeta ; \mu) \mid \mu \in[\underline{y}(\zeta), \bar{y}(\zeta)]_{r}\right)\right\} \\
& \overline{\mathcal{C}}=\sup _{\zeta \in[x-h, x+h]}\left\{\max \left(\frac{h^{4}}{12} \underline{y}^{(4)}(\zeta ; \mu), \frac{h^{4}}{12} \bar{y}^{(4)}(\zeta ; \mu) \mid \mu \in[\underline{y}(\zeta), \bar{y}(\zeta)]_{r}\right)\right\}
\end{aligned}
$$

\section{Proof}

Under the given conditions, the difference problem (15) is consistent with the boundary value problem (10) and the operator $\left[\tilde{L}_{h}\right]_{r}$ is stable.

Since 


$$
\left[\tilde{L}_{h}\right]_{r}\left(\tilde{Y}\left(x_{j} ; r\right)-\tilde{y}\left(x_{j} ; r\right)\right)=\widetilde{W}\left(x_{j} ; r\right)-\tilde{y}\left(x_{j} ; r\right)=\left[\tilde{L}_{h}\right]_{r} \tilde{Y}\left(x_{j} ; r\right)-\left[\tilde{L}_{h}\right]_{r} \tilde{y}\left(x_{j} ; r\right)=-\left[\tilde{\mathcal{T}}_{i, \pi}(\tilde{y})\right]_{r}
$$
and

$$
\tilde{Y}\left(x_{0} ; r\right)-\tilde{y}\left(x_{0} ; r\right)=\tilde{Y}\left(x_{n+1} ; r\right)-\tilde{y}\left(x_{n+1} ; r\right)=0
$$

the stability of $\left[\tilde{L}_{h}\right]_{r}$ implies that

$$
\left|\tilde{Y}\left(x_{j} ; r\right)-\tilde{y}\left(x_{j} ; r\right)\right| \leq \frac{1}{q^{*}} \max _{1 \leq j \leq n}\left[\tilde{T}_{i, \pi}(\tilde{y})\right]_{r}
$$

such that

$$
\begin{aligned}
& \left|\underline{Y}\left(x_{j} ; r\right)-\underline{y}\left(x_{j} ; r\right)\right| \leq \frac{1}{q^{*}} \sup _{1 \leq j \leq n}\left\{\min \left(\left[\underline{\mathcal{T}}_{i, \pi}(\underline{y})\right]_{r},\left[\overline{\mathcal{T}}_{i, \pi}(\bar{y})\right]\right)\right\} \\
& \left|\bar{Y}\left(x_{j} ; r\right)-\bar{y}\left(x_{j} ; r\right)\right| \leq \frac{1}{q^{*}} \sup _{1 \leq j \leq n}\left\{\max \left(\left[\underline{\mathcal{T}}_{i, \pi}(\underline{y})\right]_{r},\left[\overline{\mathcal{T}}_{i, \pi}(\bar{y})\right]\right)\right\}
\end{aligned}
$$

The desired result follows from the Lemma in [ ] .It follows from this theorem that $\left\{\left[\tilde{y}_{i}\right]_{r}\right\}_{i=0}^{n}$ is a second-order approximation to the solution $\tilde{Y}(x ; r)$ of Eq. (10).

\section{FTPBVP Linear Emden Fowler Equation}

Consider the following linear Emden Fowler FTPBVP

$$
\left\{\begin{array}{c}
\tilde{y}^{\prime \prime}(x)=\tilde{p}(x) \tilde{y}^{\prime}(x)+q(x) \tilde{y}(x)+\widetilde{w}(x), x \in\left[x_{0}, x_{n}\right] \\
y\left(x_{0}\right)=\tilde{\alpha}, y\left(x_{n}\right)=\tilde{\beta}
\end{array}\right.
$$

Where $q(x)$ are real continuous functions on $J=\left[x_{0}, x_{n}\right]$ with $\tilde{\alpha}, \widetilde{\beta} \in \tilde{E}$. Here $\tilde{p}(x)=\frac{\widetilde{\eta}}{x}$, and $\widetilde{w}(x)$ are continuous fuzzy function, $\tilde{\eta}$ is fuzzy number such that $\tilde{\eta} \in \tilde{E}$.

\subsection{The Uniqueness of the Difference Approximation}

According to Eq. (17) $\tilde{p}(x) \in \tilde{E}$ such that $\tilde{p}(x ; r)=[\underline{p}(x ; r), \bar{p}(x ; r)]$, If their exist positive constant $\left[\underline{p}^{*}, \bar{p}^{*}\right]_{r}, q^{*}$, and $\left[\underline{p}_{*}, \bar{p}_{*}\right]_{r}, q_{*}$ such that

$0 \leq\left[\underline{p}_{*}\right]_{r} \leq|\underline{p}(x ; r)| \leq\left[\underline{p}^{*}\right]_{r}, 0 \leq\left[\bar{p}_{*}\right]_{r} \leq|\bar{p}(x ; r)| \leq\left[\bar{p}^{*}\right]_{r}, 0 \leq q_{*} \leq|q(x)| \leq q^{*}$

Substituting and simplify formula (3.8) in Eq. (6.1) we have 


$$
\left\{\begin{array}{c}
h^{2}\left[\underline{L_{i}} \underline{y}_{i}\right]_{r}:-\left(1+\frac{h}{2}\left[\underline{p}_{i}\right]_{r}\right)\left[\underline{y}_{i-1}\right]_{r}+\left(2+h^{2} q_{i}\right)\left[\underline{y}_{i}\right]_{r}-\left(1-\frac{h}{2} p_{i}\right)\left[\underline{y}_{i+1}\right]_{r}=h^{2}\left[\underline{w}_{i}\right]_{r} \\
\underline{y}\left(x_{0} ; r\right)=[\underline{\alpha}]_{r}, \underline{y}\left(x_{n} ; r\right)=[\underline{\beta}]_{r} \\
h^{2}\left[\bar{L}_{i} \bar{y}_{i}\right]_{r}:-\left(1+\frac{h}{2}\left[\bar{p}_{i}\right]_{r}\right)\left[\bar{y}_{i-1}\right]_{r}+\left(2+h^{2} q_{i}\right)\left[\bar{y}_{i}\right]_{r}-\left(1-\frac{h}{2} p_{i}\right)\left[\bar{y}_{i+1}\right]_{r}=h^{2}\left[\bar{w}_{i}\right]_{r} \\
\bar{y}\left(x_{0} ; r\right)=[\bar{\alpha}]_{r}, \bar{y}\left(x_{n} ; r\right)=[\beta]_{r} \\
i=1,2, \ldots, n
\end{array}\right.
$$

Now from the above equations we can construct the following linear system in matrix form $A Y=B$ as follows:

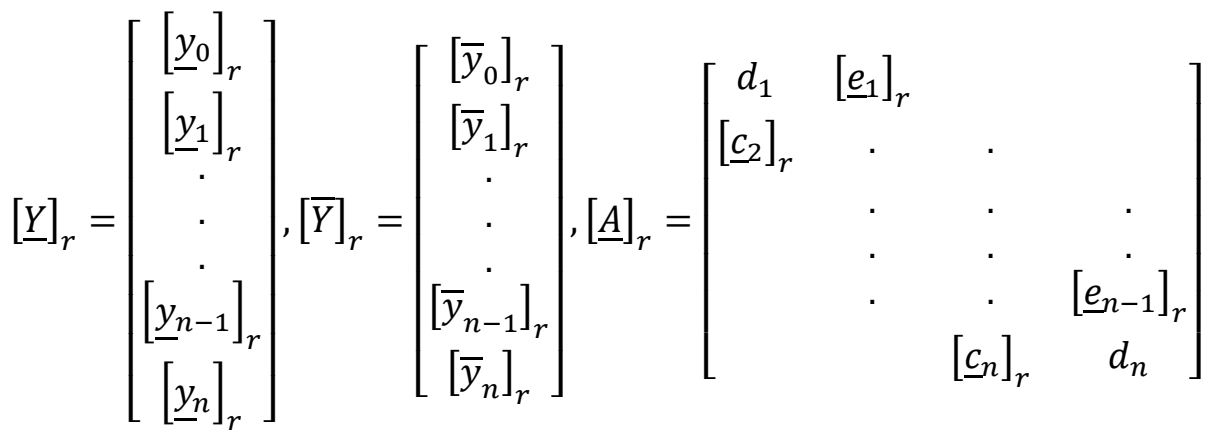

$$
\begin{aligned}
& {[\bar{A}]_{r}=\left[\begin{array}{cccc}
d_{1} & {\left[\bar{e}_{1}\right]_{r}} & & \\
{\left[\bar{c}_{2}\right]_{r}} & \cdot & \cdot & \\
& \cdot & \cdot & \cdot \\
& \cdot & \cdot & \cdot \\
& \cdot & \cdot & {\left[\bar{e}_{n-1}\right]_{r}}
\end{array}\right]} \\
& {[\underline{B}]_{r}=h^{2}\left[\begin{array}{c}
{\left[\underline{w}_{0}\right]_{r}} \\
{\left[\underline{w}_{1}\right]_{r}} \\
\cdot \\
\cdot \\
\cdot \\
{\left[\underline{w}_{n-1}\right]_{r}} \\
{\left[\underline{w}_{n}\right]_{r}}
\end{array}\right]-\left[\begin{array}{c}
{\left[\underline{c}_{2} \underline{\alpha}\right]_{r}} \\
0 \\
\cdot \\
\cdot \\
\cdot \\
0 \\
{\left[\underline{e_{n} \beta}\right]_{r}}
\end{array}\right],[\bar{B}]_{r}=h^{2}\left[\begin{array}{c}
{\left[\bar{w}_{0}\right]_{r}} \\
{\left[\bar{w}_{1}\right]_{r}} \\
\cdot \\
\cdot \\
\cdot \\
{\left[\bar{w}_{n-1}\right]_{r}} \\
{\left[\bar{w}_{n}\right]_{r}}
\end{array}\right]-\left[\begin{array}{c}
{\left[\bar{c}_{2} \bar{\alpha}\right]_{r}} \\
0 \\
\cdot \\
\cdot \\
\cdot \\
0 \\
{\left[\bar{e}_{n} \bar{\beta}\right]_{r}}
\end{array}\right]}
\end{aligned}
$$

where $[\underline{A}, \bar{A}]_{r}$ are $\mathrm{m} \times \mathrm{s}$ fuzzy matrices such that for all $r \in[0,1]$ we have $\left[\underline{c}_{i}\right]_{r}=-\left(1+\frac{h}{2}\left[\underline{p}_{i}\right]_{r}\right),\left[\bar{c}_{i}\right]_{r}=-\left(1+\frac{h}{2}\left[\bar{p}_{i}\right]_{r}\right), d_{i}=\left(2+h^{2} q_{i}\right)$, and $\left[\underline{e}_{i}\right]_{r}=-\left(1-\frac{h}{2}\left[\underline{p}_{i}\right]_{r}\right),\left[\bar{e}_{i}\right]_{r}=-\left(1-\frac{h}{2}\left[\bar{p}_{i}\right]_{r}\right)$ for $i=1,2, \ldots, n$. We prove that there is a unique $\left\{\tilde{y}_{i+1}\right\}_{i=0}^{n}$ by showing that the tri-diagonal matrix $[\tilde{A}]_{r}$ is strictly diagonally dominant and hence nonsingular.

Theorem 6.1: Suppose that the functions $\tilde{p}(x ; r)$ and $q(x)$ satisfy $(18)$ and the step size, 


$$
\begin{aligned}
& h_{1}<\inf \left\{\min \left\{\left(2 /\left[\underline{p}^{*}\right]_{r},\left[\underline{p_{*}}\right]_{r} / q^{*}\right),\left(2 /\left[\bar{p}^{*}\right]_{r},\left[\bar{p}_{*}\right]_{r} / q^{*}\right)\right\}\right\} \\
& h_{2}<\inf \left\{\max \left\{\left(2 /\left[\underline{p}^{*}\right]_{r},\left[\underline{p}^{*}\right]_{r} / q^{*}\right),\left(2 /\left[\bar{p}^{*}\right]_{r},\left[\bar{p}_{*}\right]_{r} / q^{*}\right)\right\}\right\}
\end{aligned}
$$

then the matrix $[\tilde{A}]_{r}$ is strictly diagonally dominant and hence nonsingular.

\section{Proof}

If

$$
h_{1}<\inf \left\{\min \left\{\left(2 /\left[\underline{p}^{*}\right]_{r},\left[\underline{p}_{*}\right]_{r} / q^{*}\right),\left(2 /\left[\bar{p}^{*}\right]_{r},\left[\bar{p}_{*}\right]_{r} / q^{*}\right)\right\}\right\}
$$

, then

$$
\left|\left[\underline{c}_{i}\right]_{r}\right|=\left(1+\frac{h}{2}\left[\underline{p_{i}}\right]_{r}\right), \quad\left|\left[\underline{e}_{i}\right]_{r}\right|=\left(1-\frac{h}{2}\left[\underline{p_{i}}\right]_{r}\right)
$$

and

$$
\left|\left[\underline{c}_{i}\right]_{r}\right|+\left|\left[\underline{e}_{i}\right]_{r}\right|=2<d_{i} i=1,2, \ldots, n
$$

then $\left|\left[\underline{e}_{1}\right]_{r}\right|<d_{1},\left|\left[\underline{c}_{n}\right]_{r}\right|<d_{n}$.

Similarly if

, then

$$
h_{2}<\inf \left\{\max \left\{\left(2 /\left[\underline{p}^{*}\right]_{r},\left[\underline{p}_{*}\right]_{r} / q^{*}\right),\left(2 /\left[\bar{p}^{*}\right]_{r},\left[\bar{p}_{*}\right]_{r} / q^{*}\right)\right\}\right\}
$$

and

$$
\left|\left[\bar{c}_{i}\right]_{r}\right|=\left(1+\frac{h}{2}\left[\underline{p_{i}}\right]_{r}\right), \quad\left|\left[\bar{e}_{i}\right]_{r}\right|=\left(1-\frac{h}{2}\left[\underline{p_{i}}\right]_{r}\right)
$$

$$
\left|\left[\bar{c}_{i}\right]_{r}\right|+\left|\left[\bar{e}_{i}\right]_{r}\right|=2<d_{i} i=1,2, \ldots, n
$$

then $\left|\left[\bar{e}_{1}\right]_{r}\right|<d_{1},\left|\left[\bar{c}_{n}\right]_{r}\right|<d_{n}$.

Since $h_{1}=h_{2}=h$, we conclude that

$$
\left|\left[\tilde{c}_{i}\right]_{r}\right|+\left|\left[\tilde{e}_{i}\right]_{r}\right|=2<d_{i} i=1,2, \ldots, n
$$

then $\left|\left[\tilde{e}_{1}\right]_{r}\right|<d_{1},\left|\left[\tilde{c}_{n}\right]_{r}\right|<d_{n}$.

which completes the proof. Thus Eq. (17) has unique solution. 


\subsection{Numerical Examples}

Consider the following liner Emden- Fowler FTPBVP

$$
\begin{gathered}
\tilde{y}^{\prime \prime}(x)-\frac{\widetilde{\eta}}{x} \tilde{y}^{\prime}(x)=\frac{2}{x^{2}} \tilde{y}(x), x \in[1,2] \\
y(1)=\tilde{\sigma}, y(2)=\tilde{\sigma}
\end{gathered}
$$

Where $\tilde{\sigma}$ is a triangular fuzzy number [18] having $r$-level set $[0.8+0.2 r, 1.2-0.2 r]$ and $\eta^{\tilde{y}}=[r+1,3-r]$ for all $r \in[0,1]$.

Using Maple16 package to obtain the exact solution of this problem such that:

$$
\begin{aligned}
& \underline{Y}(x ; r)=\left(\left(\left(1-2^{\frac{1}{2} r+1+\frac{1}{2} \sqrt{r^{2}+4 r-4}}\right) \frac{1}{2} r+1-\frac{1}{2} \sqrt{r^{2}+4 r-4}\right.\right. \\
& \left.+t^{\frac{1}{2} r+1+\frac{1}{2} \sqrt{r^{2}+4 r-4}}\left(2^{\frac{1}{2} r+1-\frac{1}{2} \sqrt{r^{2}+4 r-4}-1}\right)\right) \\
& (4+r)) /\left(-52^{\frac{1}{2} r+1+\frac{1}{2} \sqrt{r^{2}+4 r-4}}\right. \\
& +52^{\frac{1}{2} r+1-\frac{1}{2} \sqrt{r^{2}+4 r-4}}
\end{aligned}
$$

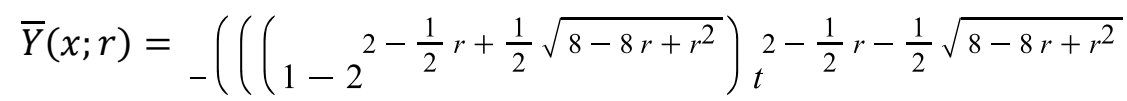

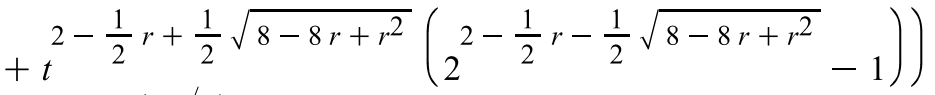

$$
\begin{aligned}
& (-6+r)) /\left(-52^{2-\frac{1}{2} r+\frac{1}{2} \sqrt{8-8 r+r^{2}}}\right. \\
& +52^{\left.2-\frac{1}{2} r-\frac{1}{2} \sqrt{8-8 r+r^{2}}\right)}
\end{aligned}
$$

$\forall r \in[0,1]$

As in the previous section it's easy to apply finite difference formula (15) on Eq. (20). For simplicity we only need one point of $1 \leq x_{\mathrm{i}} \leq 2$ to shows the exact and the numerical results for the approximate difference solution for the lower and upper bounds of Eq. (20) with step size $h=1 / 300$ and $x=1.7$ are given in the following table below:

Table 1: Difference approximate solution $\underline{y}(1.7 ; r)$ and $\bar{y}(1.7 ; r)$ of Eq. (20) at $h=1 / 100$

\begin{tabular}{ccc}
\hline$r$ & $\underline{y}(1.7 ; r)$ & $\bar{y}(1.7 ; r)$ \\
\hline 0 & 0.882872593700 & 1.325395523000 \\
0.25 & 0.938586144800 & 1.270487846000 \\
0.5 & 0.994138712700 & 1.215446825000 \\
0.75 & 1.049615481000 & 1.160280731000 \\
1 & 1.104999989000 & 1.104999989000 \\
\hline
\end{tabular}


From Table (1) one can see that the numerical results are satisfies the convex triangular fuzzy number, and for plotting these solutions with exact one we need to plot for each point $x$ such that $0 \leq r \leq 1$. For simplest illustration we plot the numerical and exact solution when $x=1.7$ as shows in the following figure:

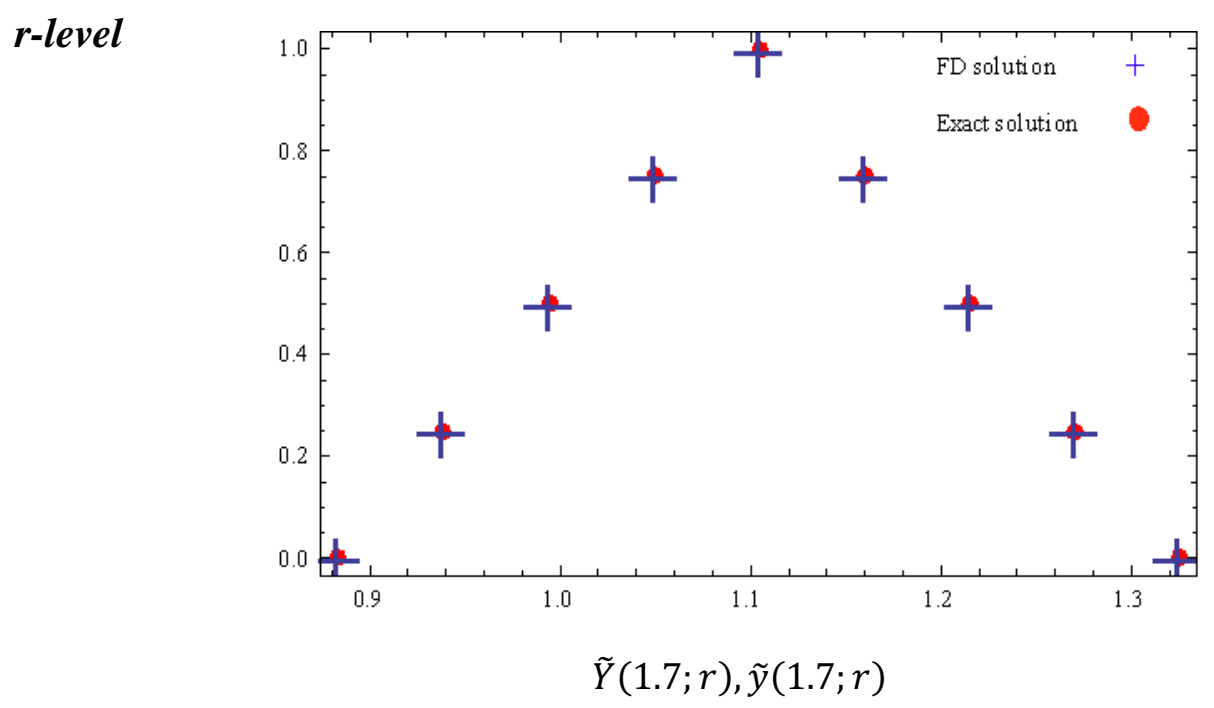

Figure 1: Exact and FD solution $\tilde{y}(1.7 ; r)$ of Eq. (20) when $h=1 / 300$

For simplest illustration of finite difference method in fuzzy environment of Eq. (20) we solved this problem at $r=0.5$ with step size $h=1 / 300$ for $1 \leq x_{i} \leq 2, i=0,2 \ldots n$ as shows in next figures and table.:
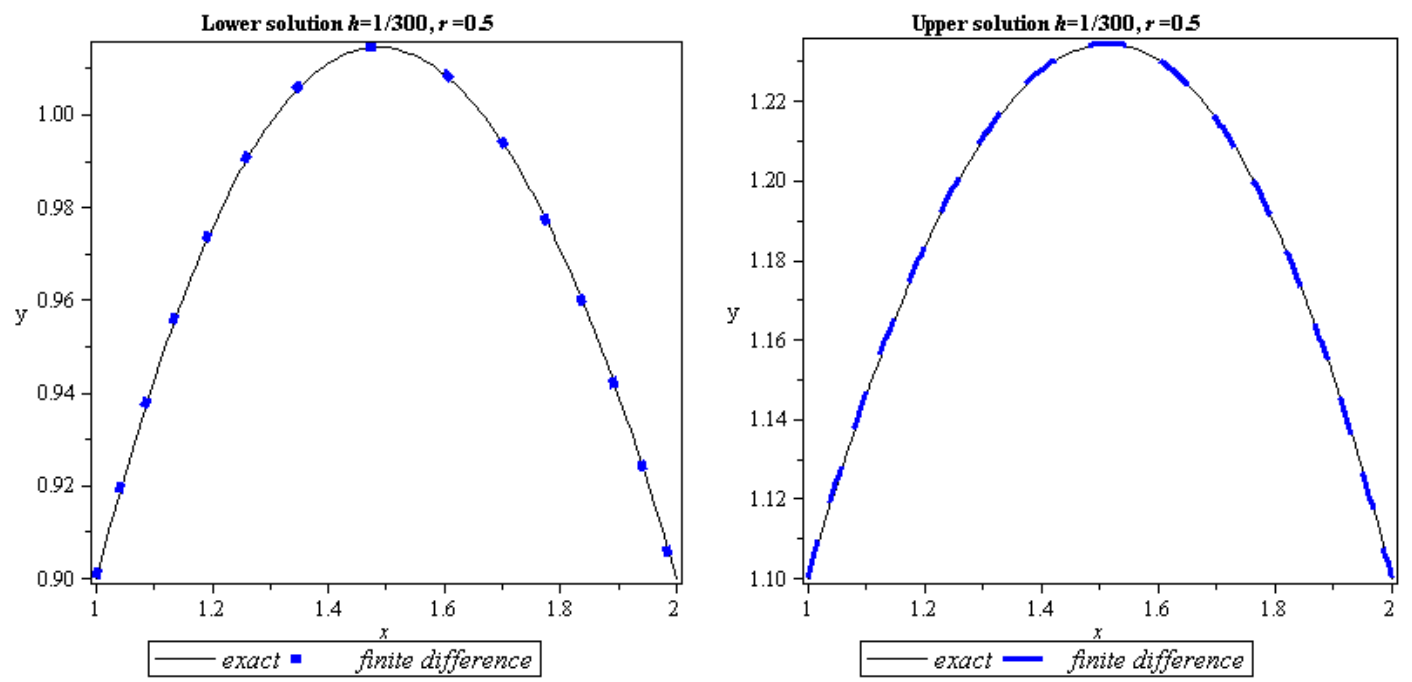

Figure 2: Difference approximate solution $\underline{y}(x ; 0.5)$ and $\bar{y}(x ; 0.5)$

Eq. (20) when $h=1 / 300$

The next table shows the absolute errors $\left[\tilde{E}_{h}\right]_{r}=\left[\underline{E}_{h}, \bar{E}_{h}\right]_{r}$ for Eq. (20) for all $x \in[0,1]$ as such that 
Table 2: Absolute error of Eq. (20) at $h=1 / 300$ and $r=0.5$.

\begin{tabular}{ccc}
\hline$x$ & {$\left[\underline{E}_{1 / 300}\right]_{0.5}$} & {$\left[\bar{E}_{1 / 300}\right]_{0.5}$} \\
\hline 1 & 0 & 0 \\
1.2 & $2.30918 \times 10-7$ & $1.39529 \times 10-7$ \\
1.4 & $3.27059 \times 10-7$ & $1.96330 \times 10-7$ \\
1.6 & $1.81888 \times 10-7$ & $3.84815 \times 10-8$ \\
1.8 & $9.09440 \times 10-7$ & $3.57208 \times 10-8$ \\
2 & 0 & 0 \\
\hline
\end{tabular}

\section{Conclusions}

In this paper, Finite Difference Method (FDM) has been successfully introduced and applied to solve 'Tow Point Fuzzy Boundary Value Problems involving linear Emden Folwer equation. Numerical examples including linear and nonlinear fuzzy initial value problems show the efficiency of implemented numerical method. The convergence and uniqueness of the difference approximation have been presented and proved.

\section{References}

[1] Z. Akbarzadeh Ghanaie, M. Mohseni Moghadam, "Solving fuzzy differential equations by RungeKutta method", The Journal of mathematics and computer science, 2(2) (2011), 208 - 221.

[2] M. Rostami, M. Kianpour, E. bashardoust, "A numerical algorithm for solving nonlinear fuzzy differential equations", The Journal of mathematics and computer science, 2(4) (2011), 667 671.

[3] R.A. Usmani, "Discrete methods for a boundary value problem with engineering applications", Math. Comput. 32(1978), 1087 - 1096.

[4] S. Ghosh, D. Roy, "Numeric-Analytic Form of the Adomian Decomposition Method for TwoPoint Boundary Value Problems in Nonlinear Mechanics", J. Eng. Mech. 133(10) (2007), 11241133.

[5] T. Ray Mahapatra, A. S. Gupta, "Heat transfer in stagnation-point flow towards a stretching sheet", Heat and Mass Transfer. 38(6) (2002), 517-52.

[6] R. Sharma, A. Ishak, I. Pop, "Partial Slip Flow and Heat Transfer over a Stretching Sheet in a Nano fluid”, (2013): http://dx.doi.org/10.1155/2013/724547.

[7] N. Kopteva, N. Madden, M. Stynes, "Grid equidistribution for reaction-diffusion problems in one dimension", Numerical Algorithms. 40(30) (2005), 305-322.

[8] N. Kopteva, M. Stynes, "A robust adaptive method for a quasilinear one-dimensional convectiondiffusion problem", SIAM J. Numer. Anal. 39 (2001), 14460-1467. 
[9] F. Schlögl, “Chemical reaction models for non-equilibrium phase transitions", Z. Physik. 253(1972), 147-161.

[10] K. Fukui, "The path of chemical reactions - the IRC approach", Chem. Res. 14 (12) (1981), 363-368.

[11] M. Goebel, U. Raitums, "Optimal control of two point boundary value problems", Control and Information Sciences. (143) (1990), 281-290.

[12] M. Popescu, "Two-point boundary value problem of control systems with parameter", CCCA. 29 (2011), 1-6.

[13] A. Omer and O. Omer, "A Pray and Pretdour Model with Fuzzy Intial Values", Hacettepe Journal of Mathematics and Statistics, 41(3) (2013), 387-395.

[14] M.S. El Naschie, “From Experimental Quantum Optics to Quantum Gravity Via a Fuzzy Kahler Manifold", Chaos Solution and Fractals, 25(2005), 969-977, 2005.

[15] M. F. Abbod, D. G Von Keyserlingk, D.A Linkens, M. Mahfouf, "Survey of Utilization of Fuzzy Technology in Medicine and Healthcare". Fuzzy sets and system, 120(2001), 331-349.

[16] Barro, R. Marin, "Fuzzy Logic in Medicine”, Heidelberg: Physica - Verlag, (2002).

[17] T. Allahviranloo, K. Khalilpour, "A Numerical Method for Two-Point Fuzzy Boundary Value Problems”, World Applied Sciences Journal 13 (10) (2011): 2137-2147.

[18] T. Allahviranloo, K. Khalilpour, "An Initial-value Method for Two-Point Fuzzy Boundary Value Problems", World Applied Sciences Journal 13 (10) (2011): 2148-2155, 2011.

[19] D. Dubois, H. Prade, "Towards fuzzy differential calculus. Part 3: Differentiation, Fuzzy Sets and Systems", 8 (1982), 225-233.

[20] S. Seikkala, “On the Fuzzy Initial Value Problem”, Fuzzy Sets and Systems. 24(3), (1987), 319330.

[21] M. Ghanbari, "Numerical Solution of Fuzzy Initial Value Problems Under Generalization Differentiability by HPM", Int.J.Industrial Mathematics 1(1) (2009), 19-39.

[22] O. S. Fard, "An Iterative Scheme for the Solution of Generalized System of Linear Fuzzy Differential Equations", World Applied Sciences Journal. 7(2009)1597-11604.

[23] H. Saberi Najafi, F. Ramezani Sasemasi, S. Sabouri Roudkoli, S. Fazeli Nodehi, "Comparison of two methods for solving fuzzy differential equations based on Euler method and Zadeh's extension", 2(2) (2011), 295 - 306.

[24] L. A. Zadeh, "The Concept of A linguistic Truth Variable and Its Application to Approximate Reasoning-I, II, III', Inform. Sci. 8 (1975),199-249.

[25] S. Sriram, P. Murugadas, "On Semiring of Intuitionstic Fuzzy Matrices", Applied Mathematical Sciences. 4 (23) (2010), 1099 - 1105.

[26] S. Siah Mansouri, N. Ahmady, "Fuzzy Differential Equation by using Characterization Theorem", communication in numerical analysis. (2012), doi:10.5899/2012/cna-00054. 